\title{
Uterine Corpus Leiomyosarcoma and Endometrial Stromal Sarcoma pN1 TNM Finding v8
}

National Cancer Institute

\section{Source}

National Cancer Institute. Uterine Corpus Leiomyosarcoma and Endometrial Stromal Sarcoma pN1 TNM Finding v8. NCI Thesaurus. Code C139841.

Uterine corpus leiomyosarcoma or endometrial stromal sarcoma with regional lymph node metastasis. (from AJCC 8th Ed.) 